VRE was isolated in $12(1.6 \%)$ of the neonates. 6 of the neonates $(50 \%)$ were born in public hospitals and $50 \%$ in private hospitals. To prevent the outbreaks in NICU we isolated the babies. Non of the babies were treated with vancomycin. The blood cultures were negative in all of them inspite of positive rectal colonisation. The diagnosis of these babies were as follows: $7 / 12$ neonatal jaundice, $2 / 12$ neonatal dehydration, $1 / 12$ urinary tract infection, $1 / 12$ bronchopneumonia, $1 / 12$ preseptal cellulitis. Median hospital stay was 10 days(3-29 days). 2/3 of newborn were born with C/S delivery, there was no hospitalisation history.

Conclusion We wanted to emphasize the uncontrolled use of antibiotics can be a problem in future therefore surveillance studies should be performed.

\section{SERUM SILICON DURING THE FIRST YEAR OF LIFE}

doi:10.1136/archdischild-2012-302724.1344

'NM Díaz-Gómez, 'E Doménech, 'E Bisse, 'F Barroso, 'LM Martin. 'Paediatrics, University of La Laguna, La Laguna, Spain; ${ }^{2}$ University of Fribourg, Fribourg, Germany

Background and Aims Serum silicon (SSi) declines with age. Silicon is known to have positive effects on bone metabolism, but SSi in preterm infants and its relationship with other oligoelements have received little attention.

To study changes in SSi levels during the first year of life in preterm infants and to determine (a) whether there are differences compared with term newborns and one-year-old healthy infants, (b) their relationship with serum zinc and copper levels.

Methods We studied:

(a) 42 preterm infants (GA: $32 \pm 1.8$ wk.; birthweight: $1651 \pm 281$ g) assessed at 36 and 40 weeks post-conceptional age (PCA) and at 12 months corrected age (CA),

(b) 30 healthy full-term newborns aged 2-3 days and

(c) 30 healthy full-term infants aged 12 months.

At each evaluation, we recorded anthropometric measurements, serum $\mathrm{Si}, \mathrm{Zn}, \mathrm{Cu}$ (atomic absorption spectrometry) and bone alkaline phosphatase (immunoradiometric assay).

Results Preterm infants showed significantly higher SSi levels than non-preterm infants in all measurements. Although SSi decreased significantly between 40 weeks PCA and 12 months CA, it remained higher than in non-preterm infants. At 40 weeks PCA, zinc levels were lower while copper and bone alkaline phosphatase were higher in preterm infants. At 12 months the differences were not significant. There were no significant correlations between serum silicon, zinc and copper concentrations in any of the groups. Conclusions SSi concentration in preterm newborns was significantly higher than in full-term newborns. Although it decreased during the first year of life, SSi remained higher than in full-term infants aged 12 months.

\section{THE VALUE OF TUBULARY PHOSPHATE REABSORPTIONRATIO IN DIAGNOSIS OF OSTEOPENIA OF PREMATURITY}

doi:10.1136/archdischild-2012-302724.1345

DB Acar, S Kavuncuoglu, E Aldemir, S Ozbek, M Cetinkaya, G Buyukkale, M Payasli, 0 Korkmaz. Kanuni Sultan Suleyman Training and Research Hospital, Istanbul, Turkey

Objective The aim of this study was to evaluate the value of tubulary phosphorus reabsorption (TPR) ratio in diagnosis of osteopenia of prematurity.

Methods This prospective study was performed between June 2009 and March 2011 and premature infants < 32 weeks of gestation and/or < 1500 gram were included. Maternal and neonatal demographic data were all recorded. The plasma Ca, P, ALP and $25-\mathrm{OH}$ vitamin $\mathrm{D}$ levels of mothers and infants were evaluated. The neonatal morbidities, duration of hospitalizati, on and total parenteral nutrition were also recorded. Infants were evaluated at postnatal 40th week. Bone mineralization was assesed by plasma $\mathrm{Ca}, \mathrm{P}$, ALP, urea, creatinine and GGT levels in combination with femur $\mathrm{X}$-ray. Also, urine was collected to determine urinary $\mathrm{Ca}$ and $\mathrm{P}$ levels and tubulary phosphate reabsorption was calculated.

Results No significant differences were detected between infants with and without osteopenia of prematurity in terms of maternal biochemical values. On the postnatal 40th week, infants with TPR $>95 \%$ had significantly higher ALP and lower P levels compared with those who had lower TPR. The sensitivity, specificity, positive predictive value and negative predictive value of TPR ratio in diagnosis of prematurity of osteopenia were found to be $27.2 \%, 82.7 \%$, $17.1 \%$ and $89.6 \%$, respectively.

Conclusion In conclusion, TPR ratio can be used as an ancillary diagnostic marker in addition to primary diagnostic tests in diagnosis of prematurity of osteopenia.

\section{HYPOTHYROXINEMIA VS HYPOTHYROIDISM IN VERY LOW BIRTH WEIGHT INFANTS}

doi:10.1136/archdischild-2012-302724.1346

${ }^{1} \mathrm{H}$ Tatar Aksoy, ${ }^{1} \mathrm{R}$ Özdemir, ${ }^{1} \mathrm{I}$ Çelik, ${ }^{1} \mathrm{O}$ Erdeve, ${ }^{2} \mathrm{U}$ Dilmen. ${ }^{1} \mathrm{NICU}$, Zekai Tahir Burak Maternity and Teaching Hospital, Department of Neonatology; 'Zekai Tahir Burak Maternity and Teaching Hospital/YIldırm Beyazit University Department of Pediatrics, Ankara, Turkey

Transient hypothyroxinemia without elevated thyroid-stimulating hormone (TSH) levels is common in prematurity, especially in verylow-birth weight (VLBW) infants. The transient hypothyroxinemia of prematurity (THOP) has been seen as a "benign" condition. Infants were classified as THOP by low thyroxine (T4) value without elevated TSH value $(<20 \mu \mathrm{IU} / \mathrm{mL})$. Primary hypothyroidism $(\mathrm{PH})$ defined by low thyroxine (T4) and elevated thyroid-stimulating hormone (TSH) levels. Both of them can be seen at premature infants.

Retrospectively we compared the premature infants born at $\leq 32$ weeks who required thyroxine supplementation for THOP and hypothyroidism. 24 neonates required thyroxine supplementation for THOP and 18 neonates for $\mathrm{PH}$ were included the study between January 2008 and December 2010.

There were no statistically differences in respect to demographic and prenatal characteristics between two groups. There was mild positive correlation between free T3, free T4 levels and gestational age. Median starting time of thyroxine supplementation was 13 days in $\mathrm{PH}$ and 21 days in THOP group $(\mathrm{p}=0.014)$. There were no statistically differences between groups in respect to birth-weight, hospitalization time, sepsis, NEC, PDA, and RDS rates. Although the THOP group started the thyroxine supplementation late, median weight of the neonates at discharge were significantly higher in THOP group (1774 vs $2070 \mathrm{p}=0,018$ ). Weight gaining per day after the thyroxine supplementation was significantly higher than the days before supplementation started ( $\mathrm{p}=0.001)$.

Infants who get enough calories but not satisfactory gaining weight should be screened for THOP and PH.

\section{BODY COMPOSITION AT 32-36 WEEKS CORRECTED AGE IN INFANTS BORN BEFORE 32 WEEKS GESTATION}

doi:10.1136/archdischild-2012-302724.1347

'MB Cumin, ${ }^{2} \mathrm{~T}$ Donovan, 'PB Colditz, 'BE Lingwood. 'University of Queensland Centre for Clinical Research, The University of Queensland; ${ }^{2}$ Grantley Stable Neonatal Unit, Royal Brisbane and Women's Hospital, Herston, OLD, Australia 
Background Preterm infants at term equivalent age are smaller than full term infants with a higher fat mass. The aim of this study was to determine if alterations in adiposity are already evident at 32-36 weeks corrected age (CA).

Methods Preterm babies $(n=21)$ born before 32 weeks gestation were studied between 32 and 36 weeks CA using the PEAPOD Infant Body Composition System to assess \% body fat. The data were compared with a second group of babies $(n=17)$ born at 32-36 weeks gestation.

Results Mean \% body fat at 32-36w CA in infants born < 32w was $14.1 \pm 5.8$ (mean \pm SD) and this was significantly higher than $\%$ body fat in infants born at 32-36 weeks $(7.9 \pm 4.5, \mathrm{P}<0.01)$. Mean \% body fat at 32-36 weeks in infants born $<32 \mathrm{w}$ was also significantly higher than \% fat at birth in infants born at term $(10.1 \pm 4.1, \mathrm{P}<0.05)$. \% body fat in infants born $<32 \mathrm{w}$ was positively correlated $(\mathrm{R}=0.59, \mathrm{P}<0.05)$ with post-natal age at measurement suggesting that longer periods of ex utero nutrition result in greater increases on \% body fat. \% body fat in infants born $<32 \mathrm{w}$ was also positively correlated with weight at the time of measurement $(R=0.56, P<0.01)$ and there was a tendency for those babies who had gained weight most rapidly since birth to have higher \% fat.

Conclusion \% body fat in infants born $<32$ weeks is elevated by 32-36 weeks CA.

\section{EARLY FORMATION OF PHOTOISOMERS DURING PHOTOTHERAPY IS NOT SIGNIFICANTLY ENHANCED BY INCREASING IRRADIANCE OR EMPLOYING PHOTODIODE LIGHTS}

doi:10.1136/archdischild-2012-302724.1348

${ }^{1} \mathrm{~K}$ Mreihil, ${ }^{2 \mathrm{P}}$ Madsen, ${ }^{1,3} \mathrm{~B}$ Nakstad, ${ }^{4} \mathrm{~F}$ Ebbesen, ${ }^{3,5} \mathrm{TW}$ R Hansen. 'Barne- og Ungdomsklinikken, Akershus University Hospital, Lørenskog, Norway; ${ }^{2}$ Pediatrics, Aalborg Sygehus Nord, Aalborg, Denmark; ${ }^{3}$ Faculty of Medicine, University of Oslo, Oslo University Hospital, Oslo, Norway; ${ }^{4}$ Pediatrics, Aalborg Sygehus Nord, Faculty of Medicine, University of Aarhus, Aalborg, Denmark: ${ }^{5}$ Kvinne- og Barne Klinikken, Oslo University Hospital Rikshospitalet, Oslo, Norway

Background Phototherapy is the mainstain of treatment for neonatal jaundice. Photoisomers are more polar than native bilirubin IX-alpha $(z, z)$ and can be excretedinbileandurine without conjugation. Rapid formation of PI may be an important component of the crash-cartapproach to extreme NJ.

Objective To compare the rate and degree of photoisomerization during intensive phototherapy using single or double banks of fluorescent lights vs a single bank of photodiodes.

Design and methods: The study was approved by the regional research ethics committee. 42 newborn infants due to receive phototherapy for $\mathrm{NJ}$ according to Norwegian national guidelines. Enrolled by written informed consent from the parents. Infants were randomly to one of 3 groups:

i. single unit phototherapy with fluorescent lights

ii. double unit fluorescent phototherapy;

iii. single unit photodiode. Irradiance was measured on the back and flanks.

Blood was drawn at $0,15,30,60,120$, and240 minutes. Total serum bilirubin was analyzed by cooximetry. Serum samples were frozen and analyzed for photoisomers by HPLC. Data were analyzed using Anova and t-tests.

Results Irradiance was significantly higher using double fluorescent lights vs single fluorescent and photodiodes (40.4 p<0.05 for double vs single fluorescent and single photodiodes, measured on the back). 2-way Anova was highly significant for increase of PI over time $(p<0.0001)$, and significant for group differences $(p<0.05)$.

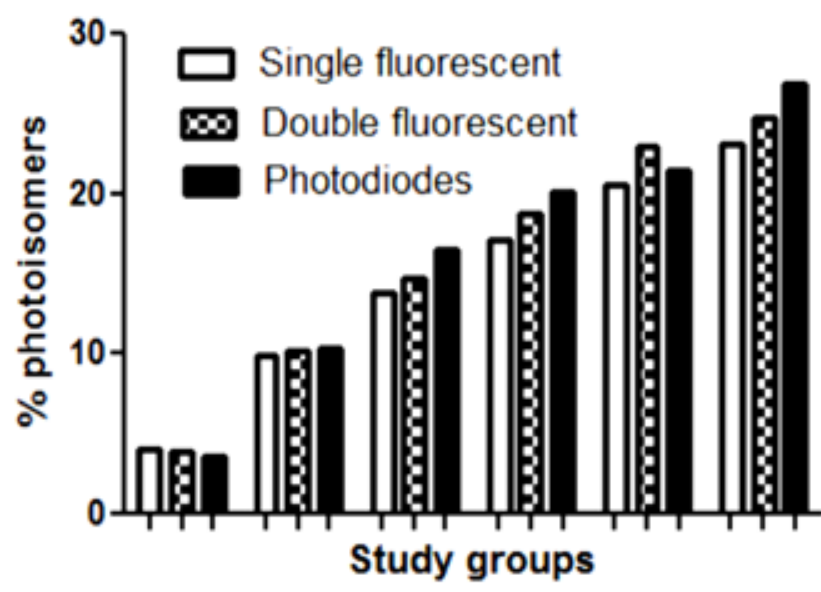

Abstract 1348 Figure 1 Study Groups

Formation of PI reached $25 \%$ at $4 \mathrm{~h}$, anddid not appear to have plateaued.

Conclusions Formation of PI is rapid during intensive phototherapy. However, increasing irradiance or chancing the character of thelight source did not significantly improve the rate or level of PI formation.

\section{TREATING INFANTS OF VITAMIN D DEFICIENT MOTHERS - IS ROUTINE SUPPLEMENTATION WITH ABIDEC ALONE SUFFICIENT}

doi:10.1136/archdischild-2012-302724.1349

A Rakhecha, K Ganesan. Department of Neonatology, St Mary's Hospital, Central Manchester University Hospitals, Manchester, UK

Background and Aims Infants with maternal vitamin D deficiency are at increased risk of low vitamin D levels. Cholecalciferol is the usual treatment for such infants. We wanted to see whether routine vitamin supplementation with Abidec alone was enough to normalize vitamin $\mathrm{D}$ levels in such infants.

Methods All infants infants with maternal vitamin $\mathrm{D}$ deficiency, after August 2011 were given $0.6 \mathrm{ml}$ Abidec which provides $400 \mathrm{IU}$ of vitamin D2. Vitamin D levels were done at birth and infants with borderline $(10-20 \mathrm{ng} / \mathrm{dl})$ or deficient $(<10 \mathrm{ng} / \mathrm{dl})$ vitamin $\mathrm{D}$ levels had repeat test at follow-up. Records of all such infants, from August to November 2011 were reviewed. The results were also compared to the period when cholecaciferol was used.

Results Total 64 patients were identified. Initial vitamin D results were obtained for 60 infants of which 16 infants were deficient (range 3.1 to $9.1 \mathrm{ng} / \mathrm{dl}$ ), 20 had borderline (range 10.3-19.8 ng/dl), 10 had suboptimal (range 20.6-29.7ng/dl) and 14 infants had normal vitamin $D$ levels.

Post treatment levels were available for 17 out of 36 infants with low or borderline levels. Of the 5 infants with initially deficient levels, 3 had normal, 1 had suboptimal and 1 had borderline vitamin D post treatment levels. 8 of the 12 infants with borderline vitamin $\mathrm{D}$ levels had normalized and 4 had suboptimal vitamin D levels post treatment.

These results were comparable to the period when cholecaliferol was used 